\title{
Distributed Optimization of Multiagent Systems in Directed Networks with Time-Varying Delay
}

\author{
Junxiu Yan and Hui Yu \\ College of Science, China Three Gorges University, Yichang 443002, China \\ Correspondence should be addressed to Hui Yu; yuhui@ctgu.edu.cn
}

Received 3 January 2017; Revised 19 February 2017; Accepted 16 March 2017; Published 29 March 2017

Academic Editor: Chaojie Li

Copyright ( 2017 Junxiu Yan and Hui Yu. This is an open access article distributed under the Creative Commons Attribution License, which permits unrestricted use, distribution, and reproduction in any medium, provided the original work is properly cited.

This paper addresses a distributed consensus optimization problem of a first-order multiagent system with time-varying delay. A continuous-time distributed optimization algorithm is proposed. Different from most ways of solving distributed optimization problem, the Lyapunov-Razumikhin theorem is applied to the convergence analysis instead of the Lyapunov-Krasovskii functionals with LMI conditions. A sufficient condition for the control parameters is obtained to make all the agents converge to the optimal solution of the system. Finally, an example is given to validate the effectiveness of our theoretical result.

\section{Introduction}

In recent years, the distributed optimization problem of multiagent systems has been investigated by many researchers; researches on distributed optimization and control theorem have been developing rapidly and have been applied to various fields of industry and defense, like smart grid $[1,2]$, sensor networks [3], social networks [4], and so on. The objective of distributed optimization problem is to solve an optimization problem cooperatively in a distributed way, where the objective function is formed by a sum of local objective functions, and each agent can only know one local objective function. The ultimate goal is to make the states of all the agents converge to optimal solution of the optimization problem via local coordination. Compared with the consensus problem of multiagent systems, which makes all agents achieve a common state [5-8], not only does the optimization problem make all agents achieve the same state, but also at the same time the achieved state minimizes the optimization problem.

The current literatures about distributed optimization problems are more focused on discrete-time algorithms (see [9-12] and references therein). In both papers $[9,11]$, discretetime subgradient algorithms are proposed for unconstrained, separable, convex optimization problem and each agent communicates with the other agents over a time-varying network topology. A projected consensus subgradient algorithm is proposed for constrained optimization problem in [10], and, in [12], the authors devise two distributed primaldual subgradient algorithms over networks with dynamically changing topologies but satisfying a standard connectivity property. But, recently, some continuous-time methods have also been successfully used to solve distributed optimization problem. Based on the gradient algorithm and integral feedback, auxiliary-variables are introduced in continuoustime dynamical system [13-15]. From the control system viewpoint, a continuous-time multiagent system dynamic is proposed with undirected communication topology [13]; the algorithm is further investigated over a strongly connected and weight balanced directed graph [16], and even a modified system is proposed in [14] with auxiliary-variables no longer needing to exchange information. In [17], the authors present a second-order multiagent system for distributed optimization network under bound constraints, and, in [18], a distributed protocol design for the high-order agent-network under a connected communication topology is proposed. In order to avoid using auxiliary-variables, a family of ZeroGradient-Sum algorithms are proposed over fixed communication topology in [19].

On the other hand, it is common that time-delay exists in practical systems because of the finite speeds of information transmission and spreading as well as traffic 
congestions. Therefore, time-delay should be taken into account in algorithm design of multiagent systems. For timedelay systems modelled by delayed differential equations, an effective way to deal with their convergence and stability analysis is based on the Lyapunov-Krasovskii functionals or Lyapunov-Razumikhin functions. Most of the existing works concentrate on Lyapunov functions combining with Linear Matrix Inequality (LMI) techniques to deal with the consensus problem of multiagent systems with time-delay [20, 21]. The methods based on Lyapunov-Krasovskii functionals can be applied to a wide variety of problems and may provide necessary and sufficient conditions of convergence and stability, but it often leads to computational complexity and poor scalability. When the number of the agents is large, it would be difficult to verify the solvability of the LMI conditions. However, based on the Lyapunov-Razumikhin theorem, the authors propose a neighbor-based distributed controller $[7,8]$ enabling the agents to achieve consensus along with interconnection delays, which can avoid verifying the LMI condition and reducing computational burden. In [15], distributed consensus optimization algorithms are proposed for continuous-time multiagent systems with timedelay, and some sufficiency conditions based on LMI are obtained.

Motivated by the above observations, the distributed consensus optimization problem of continuous-time multiagent systems with time-varying delay is considered. The interconnected graph is assumed to be directed, strongly connected, and weighted-balanced. The Lyapunov-Razumikhin function is used in the stability analysis. The convergence of the proposed algorithm is guaranteed with the model parameters satisfying some conditions. Meanwhile, the conditions can also give an estimate of the upper bound of the time-delay, which can avoid verifying and calculating the complicated LMI conditions. From the results, we can also see clearly the relationship among the parameters in the system.

The outline of this paper is organized as follows. Some basic knowledge on the algebraic graph theory and useful lemmas are presented in Section 2. The convergence results of the algorithm are established under the given communication condition on network topology by applying LyapunovRazumikhin Theorem in Section 3. An example is provided to illustrate the result in this paper in Section 4. Finally, the concluding remarks are given in Section 5.

Notations. $\mathscr{R}$ and $\mathscr{R}^{n}$ represent the set of real numbers and the set of $n \times 1$ real vectors, respectively; $I_{n} \in \mathscr{R}^{n \times n}$ is the $n \times n$ identity matrix; $\mathbf{1}_{n}$ (or $\mathbf{0}_{n}$ ) denotes an $n$ dimensional column vector whose all entries being 1 (or 0 ); $A^{T}$ represents the transpose of a matrix $A$; for vectors $x_{1}, x_{2}, \ldots, x_{n}$, $\operatorname{col}\left(x_{1}, x_{2}, \ldots, x_{n}\right)=\left[x_{1}^{T}, x_{2}^{T}, \ldots, x_{n}^{T}\right]^{T}$; for a vector $w$, then $\|w\|=\sqrt{w^{T} w}$ represents the standard Euclidean norm.

\section{Preliminaries and Problem Statement}

2.1. Preliminaries. Consider a multiagent system consisting of $N$ agents, if each agent is regarded as a node, the communication topology among these agents can be described by a weighted digraph $\mathscr{G}=(\mathscr{V}, \mathscr{E}, \mathscr{A})$ with the finite set of nodes $\mathscr{V}=\{1,2, \ldots, N\}$ and edge set $\mathscr{E} \subset \mathscr{V} \times \mathscr{V}$. An edge starts from $i$ and ends on $j$, which means that agent $i$ can send information to agent $j$. The weighted adjacency matrix $\mathscr{A}=\left[a_{i j}\right] \in R^{N \times N}$ is defined as $a_{i j}>0$ if $(i, j) \in \mathscr{E}$ and $a_{i j}=0$ otherwise. If $\sum_{j=1}^{N} a_{i j}=\sum_{j=1}^{N} a_{j i}$ for all $i \epsilon$ $\mathscr{V}$, the digraph $\mathscr{G}$ is called weighted-balanced. A path is a sequence of connected edges in a graph. If there is a path between any two nodes of a digraph $\mathscr{G}$, then digraph $\mathscr{G}$ is said to be strongly connected, otherwise disconnected. The degree matrix $\mathscr{D}=\operatorname{diag}\left\{d_{1}, d_{2}, \ldots, d_{N}\right\} \in \mathscr{R}^{N \times N}$ of graph $\mathscr{G}$ is a diagonal matrix with the $i$ th diagonal element being $d_{i}=\sum_{j=1}^{N} a_{i j}$ for $i \in \mathscr{V}$. The Laplacian of graph $\mathscr{G}$ is defined as $L=\mathscr{D}-\mathscr{A}$.

The next lemmas related to the important properties of Laplace $L$ and provide useful mathematical tools.

Lemma 1 (see [22]). Laplace matrix L has at least one zero eigenvalue with $\mathbf{1}_{N}=[1,1, \ldots, 1] \in \mathscr{R}^{N}$ as its eigenvector, and all the nonzero eigenvalues of $L$ have positive real parts. Laplacian $L$ has a simple zero eigenvalue if and only if $\mathscr{G}$ is strongly connected.

Lemma 2. For matrices $A, B, C$ and $D$ with appropriate dimensions, the Kronecker product $\otimes$ satisfies $(1)(A \otimes B)(C \otimes$ $D)=(A C) \otimes(B D) ;(2)(A \otimes B)^{T}=A^{T} \otimes B^{T} ;(3)(A \otimes B)^{-1}=$ $A^{-1} \otimes B^{-1}$.

Lemma 3 (see [23]). For a given real matrix $S=\left(\begin{array}{cc}X & Y \\ Y^{T} & Z\end{array}\right)$ with $X^{T}=X$ and $Z^{T}=Z$, then the following conditions are equivalent:

(1) $S>0$;

(2) $X>0, Z-Y^{T} X^{-1} Y>0$;

(3) $Z>0, X-Y X^{-1} Y^{T}>0$.

2.2. Problem Statement. We consider a multiagent system consisting of $N$ agents. The dynamics of the $i$ th agent, $i \in \mathscr{V}$, is described by

$$
\dot{x}_{i}(t)=u_{i}(t),
$$

where $x_{i} \in \mathscr{R}^{m}$ denotes the state of agent $i$ and $u_{i} \in \mathscr{R}^{m}$ is the control input.

Consider the multiagent optimization problem, in which the goal is to minimize the sum of local cost functions associated with the individual agent. More specially, it can be expressed as

$$
\text { minimize } f(x)=\sum_{i=1}^{N} f_{i}(x), \quad x \in \mathscr{R}^{m} .
$$

Let $\mathbf{x}=\operatorname{col}\left(x_{1}, x_{2}, \ldots, x_{N}\right) \in \mathscr{R}^{N m}$. Next, we provide an alternative formulation of (2), that is,

$$
\begin{array}{ll}
\text { minimize } & f(\mathbf{x})=\sum_{i=1}^{N} f_{i}\left(x_{i}\right), \quad x_{i} \in \mathscr{R}^{m}, \\
\text { subject to } & \left(L \otimes I_{m}\right) \mathbf{x}=0_{N m} .
\end{array}
$$


We can see that the problem (2) on $\mathscr{R}^{m}$ is equivalent to the problem (3) on $\mathscr{R}^{N m}$.

In this paper, our goal is to design a distributed controller for each agent such that the states of all the agents converge to the optimal solution of the optimization problem (2) via local communication.

Before proceeding, we give the following assumption on the local cost function $f_{i}$ based on convex analysis [24].

Assumption 4. (a) For each $i \in \mathscr{V}, f_{i}$ is differentiable and its gradient is Lipschitz with constant $\rho_{i}>0$ in $\mathscr{R}^{m}$ :

$$
\left\|\nabla f_{i}(x)-\nabla f_{i}(y)\right\| \leq \rho_{i}\|x-y\|, \quad \forall x, y \in \mathscr{R}^{m} .
$$
0

(b) for $i \in \mathscr{V}, f_{i}$ is $m_{i}$-strongly convex with constant $m_{i}>$

$$
\begin{aligned}
&(x-y)^{T}\left(\nabla f_{i}(x)-\nabla f_{i}(y)\right) \geq m_{i}\|x-y\|^{2}, \\
& \forall x, y \in \mathscr{R}^{m} .
\end{aligned}
$$

Remark 5. Under Assumption 4(b), we can note that $f$ is strictly convex; then the problem (3) has an unique optimal solution.

Assumption 6. The digraph $\mathscr{G}$ is weighted-balanced and strongly connected.

From Lemma 1 and Assumption 6, there exists a matrix $Q \in \mathscr{R}^{N \times(N-1)}$ with

$$
\begin{aligned}
\mathbf{1}_{N}^{T} Q & =0, \\
Q^{T} Q & =I_{N-1}, \\
Q^{T} & =I_{N}-\frac{1}{N} \mathbf{l}_{N} \mathbf{1}_{N}^{T},
\end{aligned}
$$

such that the matrix $Q^{T} L Q=H$, where the real parts of all the eigenvalues of $H$ are positive, and $H+H^{T}$ is positive definite.

When considering the presence of time-varying communication delay among the information transmission, the continuous-time distributed optimization protocol is proposed for agent $i(i \in \mathscr{V})$ as follows:

$$
\begin{aligned}
& u_{i}(t)=-k \sum_{j=1}^{N} a_{i j}\left[x_{i}(t-\tau(t))-x_{j}(t-\tau(t))\right]-w_{i}(t) \\
& -\gamma \nabla f_{i}\left(x_{i}(t)\right), \\
& \dot{w}_{i}(t)=\alpha \sum_{j=1}^{N} a_{i j}\left[x_{i}(t-\tau(t))-x_{j}(t-\tau(t))\right] \\
& w_{i}(0)=0,
\end{aligned}
$$

where $w_{i}(t)$ is an auxiliary state of agent $i$ and $\tau(t)$ is a continuously differentiable function satisfying $\tau(t) \in[0, \tau]$ with $\tau>0$ for all $t>0$ and $k, \alpha, \gamma$ are the scalar tuning positive parameters; $-\gamma \nabla f_{i}\left(x_{i}(t)\right)$ is the gradient term to guide the agents for optimization; $-k \sum_{j=1}^{N} a_{i j}\left[x_{i}(t-\tau(t))-x_{j}(t-\tau(t))\right]$ is the consensus term with time-delay to make all the agents converge to the same point; $-w_{i}(t)$ is an integral term to correct the error caused by the consensus term.

Let

$$
\begin{aligned}
& \mathbf{w}(t)=\operatorname{col}\left(w_{1}(t), w_{2}(t), \ldots, w_{N}(t)\right), \\
& \nabla \bar{f}(x(t)) \\
& \quad=\operatorname{col}\left(\nabla f_{1}\left(x_{1}(t)\right), \nabla f_{2}\left(x_{2}(t)\right), \ldots, \nabla f_{N}\left(x_{N}(t)\right)\right) .
\end{aligned}
$$

Then the closed-loop system of (1) and (7) can be expressed as a compact form:

$$
\begin{aligned}
\dot{\mathbf{x}}(t) & =-k\left(L \otimes I_{m}\right) \mathbf{x}(t-\tau(t))-\mathbf{w}(t)-\gamma \nabla \bar{f}(\mathbf{x}(t)), \\
\dot{\mathbf{w}}(t) & =\alpha\left(L \otimes I_{m}\right) \mathbf{x}(t-\tau(t)) .
\end{aligned}
$$

Let the right-side of closed-loop system (9) be equal to 0; then we can get the equilibrium point $\left(\mathbf{x}^{*}, \mathbf{w}^{*}\right)$, that is,

$$
\begin{aligned}
-k\left(L \otimes I_{m}\right) \mathbf{x}^{*}-\mathbf{w}^{*}-\gamma \nabla \bar{f}\left(\mathbf{x}^{*}\right) & =0, \\
\alpha\left(L \otimes I_{m}\right) \mathbf{x}^{*} & =0 .
\end{aligned}
$$

According to the properties of Laplacian matrix and from (10), one can obtain

$$
\begin{aligned}
& \mathbf{x}^{*}=\mathbf{1}_{N} \otimes \pi, \quad \pi \in \mathscr{R}^{m}, \\
& \mathbf{w}^{*}=-\gamma \nabla \bar{f}\left(\mathbf{x}^{*}\right) .
\end{aligned}
$$

Under Assumption 6, we have $\mathbf{1}_{N}^{T} L=0$. Left multiplying the second equation of (9) by $\mathbf{1}_{N}^{T} \otimes I_{m}$ and using initial conditions $w_{i}(0)=0$, we obtain $\sum_{j=1}^{N} \dot{w}_{j}(t)=0$; then

$$
\sum_{j=1}^{N} w_{j}(t)=\sum_{j=1}^{N} w_{j}(0)=0, \quad \forall t \geq 0 .
$$

Left multiplying the second equation of (11) by $\mathbf{1}_{N}^{T} \otimes I_{m}$ again results in

$$
\begin{aligned}
0 & =\sum_{j=1}^{N} w_{j}^{*}=-\gamma\left(\mathbf{1}_{N}^{T} \otimes I_{m}\right) \nabla \bar{f}\left(\mathbf{x}^{*}\right)=-\gamma \sum_{j=1}^{N} \nabla f_{i}(\pi) \\
& =-\gamma \nabla f\left(\mathbf{x}^{*}\right) .
\end{aligned}
$$

Thus, the optimal condition $\nabla f\left(\mathbf{x}^{*}\right)=0$ is satisfied, which means $\mathbf{x}^{*}=\mathbf{1}_{N} \otimes x^{*}, x^{*} \in \mathscr{R}^{m}$ is the optimal solution of the optimization problem (3).

Using the transformation

$$
\begin{aligned}
\overline{\mathbf{x}}(t) & =\mathbf{x}(t)-\mathbf{x}^{*}, \\
\overline{\mathbf{w}}(t) & =\mathbf{w}(t)-\mathbf{w}^{*},
\end{aligned}
$$

one can shift the equilibrium point into the origin; then the system (9) can be transformed into the following form:

$$
\begin{aligned}
\dot{\overline{\mathbf{x}}}(t) & =-k\left(L \otimes I_{m}\right) \overline{\mathbf{x}}(t-\tau(t))-\overline{\mathbf{w}}(t)-\gamma \Psi(\overline{\mathbf{x}}(t)), \\
\dot{\overline{\mathbf{w}}}(t) & =\alpha\left(L \otimes I_{m}\right) \overline{\mathbf{x}}(t-\tau(t)),
\end{aligned}
$$

where $\Psi(\overline{\mathbf{x}}(t))=\nabla \bar{f}(\mathbf{x}(t))-\nabla \bar{f}\left(\mathbf{x}^{*}\right)$. 


\section{Main Results}

Before analyzing the consensus and optimization problem (9), we introduce the stability of time-delay systems. Consider the following time-delay system:

$$
\begin{gathered}
\dot{x}=f\left(t, x_{t}\right), \quad t>t_{0}, \\
x(\theta)=\varphi(\theta), \quad \theta \in\left[-\tau, t_{0}\right],
\end{gathered}
$$

where $x_{t}(\theta)=x(t+\theta), \forall \theta \in\left[-\tau, t_{0}\right]$ and $f(t, 0)=0$. In the sequel, suppose that $t_{0}=0$. Let $C\left([-\tau, 0], \mathscr{R}^{n}\right)$ be a Banach space of continuous function defined on an interval $[-\tau, 0]$, taking values in $\mathscr{R}^{n}$ with topology of uniform convergence, and with a norm $\|\varphi\|_{c}=\max _{\theta \in\left[-\tau, t_{0}\right]}\|\varphi(\theta)\|$.

The definition of the stability of the solution $x=0$ is given as follows in terms of the solution of the delayed equation (16).

Lemma 7 (see [25]). Let $\phi_{1}, \phi_{2}$, and $\phi_{3}$ be continuous, nonnegative, nondecreasing function with $\phi_{1}(s)>0, \phi_{2}(s)>$ $0, \phi_{3}(s)>0$ for $s>0$ and $\phi_{1}(0)=\phi_{2}(0)=0$. For system (16), suppose that the function $f: \mathscr{R} \times C\left([-\tau, 0], \mathscr{R}^{n}\right) \rightarrow \mathscr{R}$ takes bounded sets of $C\left([-\tau, 0], \mathscr{R}^{n}\right)$ in bounded sets of $R^{n}$. There is a continuous function $V(t, x)$ such that

$$
\phi_{1}(\|x\|) \leq V(t, x) \leq \phi_{2}(\|x\|), \quad t \in \mathscr{R}, x \in \mathscr{R}^{n} .
$$

In addition, there exists a continuous nondecreasing function $\phi(s)$ with $\phi(s)>s, s>0$ such that

$$
\left.\dot{V}(t, x)\right|_{(16)} \leq-\phi_{3}(s)
$$

If

$$
V(t+\theta, x(t+\theta))<\phi(V(t, x)), \quad \theta \in[-\tau, 0],
$$

then the solution $x=0$ of system (16) is uniformly asymptotically stable.

Usually, $V(t, x)$ is called Lyapunov-Razumikhin function if it satisfies (17) and (18) in Lemma 7.

Then the main results can be obtained as follows.

Theorem 8. Suppose Assumptions 4 and 6 hold, satisfy

$$
2 \gamma \underline{m} \geq \gamma^{2} \bar{\rho}^{2}+\underline{\lambda}_{2}
$$

and take

$$
k>k^{*}=\frac{2}{\underline{\lambda}_{1}}+\alpha
$$

and assume that

$$
\begin{aligned}
& \tau<\tau^{*} \\
& =\frac{\underline{\lambda}_{2}}{(k-\alpha)\left[(k+\alpha) \bar{\lambda}_{1}+k^{3} \bar{\lambda}_{2}\right]+\left(2 q+\delta \gamma^{2} \bar{\rho}^{2} q\right)(\bar{\mu}+1)},
\end{aligned}
$$

where $q>1, \bar{\mu}=1+k / \alpha$, and $\delta=\lambda_{\max }\left(P_{1}\right) / \lambda_{\min }(P)$, and, respectively,

$$
\begin{aligned}
& \underline{\lambda}_{1}=\lambda_{\min }\left(H^{T}+H\right), \\
& \underline{\lambda}_{2}=\lambda_{\min }\left(R-I_{2 N-2}\right) ; \\
& \bar{\lambda}_{1}=\lambda_{\max }\left(H H^{T}\right), \\
& \bar{\lambda}_{2}=\lambda_{\max }\left(H^{2}\left(H^{2}\right)^{T}\right),
\end{aligned}
$$

where $\lambda_{\min }(\cdot)$ and $\lambda_{\max }(\cdot)$ denote the smallest and the largest nonzero eigenvalue of positive semidefinite matrix, respectively.

Then, the optimization problem (3) for multiagent system (1) can be solved by the optimization control (7), where

$$
R=\left(\begin{array}{cc}
(k-\alpha)\left(H^{T}+H\right) & I_{N-1} \\
I_{N-1} & 2 I_{N-1}
\end{array}\right) \otimes I_{m} .
$$

Proof. Let

$$
\begin{aligned}
e(t) & =\left(T^{T} \otimes I_{m}\right) \overline{\mathbf{x}}(t), \\
\vartheta(t) & =\left(T^{T} \otimes I_{m}\right) \overline{\mathbf{w}}(t), \\
T & =\left[\frac{\mathbf{1}_{N}}{\sqrt{N}} Q\right] .
\end{aligned}
$$

Denote $e=\operatorname{col}\left(e_{1}, e_{2}\right)$, and $\vartheta=\operatorname{col}\left(\vartheta_{1}, \vartheta_{2}\right)$ with $e_{1}, \vartheta_{1} \in$ $\mathscr{R}^{m}$, and $e_{2}, \vartheta_{2} \in \mathscr{R}^{m(N-1)}$. By the structure of $T$ and (6), we can know that $T$ is an orthogonal matrix. Then the system (15) can be rewritten as

$$
\begin{aligned}
\dot{e}_{1}(t)= & -\gamma\left(\frac{\mathbf{1}_{N}^{T}}{\sqrt{N}} \otimes I_{m}\right) \Psi(\overline{\mathbf{x}}(t)), \\
\dot{e}_{2}(t)= & -k\left(H \otimes I_{m}\right) e_{2}(t-\tau(t))-\vartheta_{2}(t) \\
& -\gamma\left(Q^{T} \otimes I_{m}\right) \Psi(\overline{\mathbf{x}}(t)), \\
\dot{\vartheta}_{1}(t)= & 0, \\
\dot{\vartheta}_{2}(t)= & \alpha\left(H \otimes I_{m}\right) e_{2}(t-\tau(t)) .
\end{aligned}
$$

Let $\boldsymbol{\varepsilon}(t)=\operatorname{col}(e(t), \vartheta(t))=\operatorname{col}\left(e_{1}(t), e_{2}(t), \vartheta_{1}(t), \vartheta_{2}(t)\right)$, and construct the Lyapunov-Razumikhin function as

$$
V(\boldsymbol{\varepsilon}(t))=\boldsymbol{\varepsilon}^{T}(t) P \boldsymbol{\varepsilon}(t)
$$

with

$$
P=\left(\begin{array}{cccc}
1 & 0 & 1 & 0 \\
0 & I_{N-1} & 0 & I_{N-1} \\
1 & 0 & \frac{k}{\alpha} & 0 \\
0 & I_{N-1} & 0 & \frac{k}{\alpha} I_{N-1}
\end{array}\right) \otimes I_{m}
$$

We can have the fact that $P=\left(\begin{array}{cc}I_{N} & I_{N} \\ I_{N} & (k / \alpha) I_{N}\end{array}\right) \otimes I_{m}$ is positive definite since $k>\alpha$. 
The derivation of $V$ along the system (26) is given by

$$
\begin{aligned}
\dot{V}(\boldsymbol{\varepsilon}(t))= & 2 \boldsymbol{\varepsilon}^{T}(t) P \dot{\boldsymbol{\varepsilon}}(t) \\
= & 2\left[e_{1}(t)+\vartheta_{1}(t)\right]^{T} \dot{e}_{1}(t) \\
& +2\left[e_{2}(t)+\vartheta_{2}(t)\right]^{T} \dot{e}_{2}(t) \\
& +2\left[e_{1}(t)+\frac{k}{\alpha} \vartheta_{1}(t)\right]^{T} \dot{\vartheta}_{1}(t) \\
& +2\left[e_{2}(t)+\frac{k}{\alpha} \vartheta_{2}(t)\right]^{T} \dot{\vartheta}_{2}(t) .
\end{aligned}
$$

Combining the third equation of (26) and (12) gives $\vartheta_{1}(t)=$ $0, \forall t \geq 0$; then

$$
\begin{aligned}
\dot{V}(\boldsymbol{\varepsilon}(t))= & 2 e_{1}^{T}(t) \dot{e}_{1}(t)+2\left[e_{2}(t)+\vartheta_{2}(t)\right]^{T} \dot{e}_{2}(t) \\
& +2\left[e_{2}(t)+\frac{k}{\alpha} \vartheta_{2}(t)\right]^{T} \dot{\vartheta}_{2}(t) \\
= & 2 e_{1}^{T}(t) \dot{e}_{1}(t)+2 \varepsilon_{2: N}^{T}(t) P_{1} \dot{\varepsilon}_{2: N}(t),
\end{aligned}
$$

where $\varepsilon_{2: N}(t)=\operatorname{col}\left(e_{2}^{T}(t), \vartheta_{2}^{T}(t)\right), P_{1}=\left(\begin{array}{cc}I_{N-1} & I_{N-1} \\ I_{N-1} & (k / \alpha) I_{N-1}\end{array}\right) \otimes I_{m}$.

For the second and fourth equalities of system (26), we have a compact form

$$
\dot{\boldsymbol{\varepsilon}}_{2: N}(t)=C \boldsymbol{\varepsilon}_{2: N}(t)+E \boldsymbol{\varepsilon}_{2: N}(t-\tau(t))+F
$$

with $C=\left(\begin{array}{cc}0 & -I_{N-1} \\ 0 & 0\end{array}\right) \otimes I_{m}, E=\left(\begin{array}{cc}-k H & 0 \\ \alpha H & 0\end{array}\right) \otimes I_{m}$, and $F=$ $\left(\begin{array}{c}-\gamma\left(Q^{T} \otimes I_{m}\right) \Psi(\overline{\mathbf{x}}(t)) \\ 0\end{array}\right)$.

By the Leibniz-Newton formula

$$
\begin{aligned}
\boldsymbol{\varepsilon}_{2: N}(t-\tau(t))= & \boldsymbol{\varepsilon}_{2: N}(t)-\int_{t-\tau(t)}^{t} \dot{\boldsymbol{\varepsilon}}_{2: N}(s) d s \\
= & \boldsymbol{\varepsilon}_{2: N}(t)-C \int_{-\tau(t)}^{0} \boldsymbol{\varepsilon}_{2: N}(t+s) d s \\
& -E \int_{-2 \tau(t)}^{-\tau(t)} \boldsymbol{\varepsilon}_{2: N}(t+s) d s \\
& -\int_{-\tau(t)}^{0} F(t+s) d s .
\end{aligned}
$$

Therefore, the system (31) can be rewritten as

$$
\begin{aligned}
\dot{\boldsymbol{\varepsilon}}_{2: N}(t)= & \bar{F} \boldsymbol{\varepsilon}_{2: N}(t)-E C \int_{-\tau(t)}^{0} \boldsymbol{\varepsilon}_{2: N}(t+s) d s \\
& -E^{2} \int_{-2 \tau(t)}^{-\tau(t)} \boldsymbol{\varepsilon}_{2: N}(t+s) d s \\
& -E \int_{-\tau(t)}^{0} F(t+s) d s+F
\end{aligned}
$$

where $\bar{F}=E+C$.
Thus, we can get

$$
\begin{aligned}
2 \boldsymbol{\varepsilon}_{2: N}^{T} & (t) P_{1} \dot{\boldsymbol{\varepsilon}}_{2: N}(t) \\
= & \boldsymbol{\varepsilon}_{2: N}^{T}(t)\left(\bar{F}^{T} P_{1}+P_{1} \bar{F}\right) \boldsymbol{\varepsilon}_{2: N}(t) \\
& -2 \boldsymbol{\varepsilon}_{2: N}^{T}(t) P_{1} E C \int_{-\tau(t)}^{0} \boldsymbol{\varepsilon}_{2: N}(t+s) d s \\
& -2 \boldsymbol{\varepsilon}_{2: N}^{T}(t) P_{1} E^{2} \int_{-2 \tau(t)}^{-\tau(t)} \boldsymbol{\varepsilon}_{2: N}(t+s) d s \\
& -2 \boldsymbol{\varepsilon}_{2: N}^{T}(t) P_{1} E \int_{-\tau(t)}^{0} F(t+s) d s \\
& +2 \boldsymbol{\varepsilon}_{2: N}^{T}(t) P_{1} F .
\end{aligned}
$$

Combining (30) and (34) gives

$$
\begin{aligned}
\dot{V}(\boldsymbol{\varepsilon}(t))= & \boldsymbol{\varepsilon}_{2: N}^{T}(t)\left(\bar{F}^{T} P_{1}+P_{1} \bar{F}\right) \boldsymbol{\varepsilon}_{2: N}(t) \\
& -2 \boldsymbol{\varepsilon}_{2: N}^{T}(t) P_{1} E C \int_{-\tau(t)}^{0} \boldsymbol{\varepsilon}_{2: N}(t+s) d s \\
& -2 \boldsymbol{\varepsilon}_{2: N}^{T}(t) P_{1} E^{2} \int_{-2 \tau(t)}^{-\tau(t)} \boldsymbol{\varepsilon}_{2: N}(t+s) d s \\
& -2 \boldsymbol{\varepsilon}_{2: N}^{T}(t) P_{1} E \int_{-\tau(t)}^{0} F(t+s) d s \\
& +2 \boldsymbol{\varepsilon}_{2: N}^{T}(t) P_{1} F+2 e_{1}^{T}(t) \dot{e}_{1}(t) .
\end{aligned}
$$

Note that $2 a^{T} b \leq a^{T} \Phi a+b^{T} \Phi^{-1} b$ holds for any appropriate positive definite matrix $\Phi$; then let $a^{T}=-\boldsymbol{\varepsilon}_{2: N}^{T}(t) P_{1} E C, b=$ $\boldsymbol{\varepsilon}_{2: N}(t+s)$, and $\Phi=P_{1}^{-1}$; one can obtain

$$
\begin{aligned}
- & 2 \boldsymbol{\varepsilon}_{2: N}^{T}(t) P_{1} E C \int_{-\tau(t)}^{0} \boldsymbol{\varepsilon}_{2: N}(t+s) d s \\
= & \int_{-\tau(t)}^{0} 2\left(-\boldsymbol{\varepsilon}_{2: N}^{T}(t) P_{1} E C\right) \boldsymbol{\varepsilon}_{2: N}(t+s) d s \\
\leq & \tau(t) \boldsymbol{\varepsilon}_{2: N}^{T}(t) P_{1} E C P_{1}^{-1}\left(P_{1} E C\right)^{T} \boldsymbol{\varepsilon}_{2: N}(t) \\
& +\int_{-\tau(t)}^{0} \boldsymbol{\varepsilon}_{2: N}^{T}(t+s) P_{1} \boldsymbol{\varepsilon}_{2: N}(t+s) d s \\
\leq & \tau \boldsymbol{\varepsilon}_{2: N}^{T}(t) P_{1} E C P_{1}^{-1}\left(P_{1} E C\right)^{T} \boldsymbol{\varepsilon}_{2: N}(t) \\
& +\int_{-\tau(t)}^{0} \boldsymbol{\varepsilon}_{2: N}^{T}(t+s) P_{1} \boldsymbol{\varepsilon}_{2: N}(t+s) d s .
\end{aligned}
$$


Similarly, let $a^{T}=-\boldsymbol{\varepsilon}_{2: N}^{T}(t) P_{1} E^{2}, b=\varepsilon_{2: N}(t+s)$, and $\Phi=P_{1}^{-1}$; we have

$$
\begin{aligned}
-2 \boldsymbol{\varepsilon}_{2: N}^{T}(t) P_{1} E^{2} \int_{-2 \tau(t)}^{-\tau(t)} \boldsymbol{\varepsilon}_{2: N}(t+s) d s \\
=\int_{-2 \tau(t)}^{-\tau(t)} 2\left(-\boldsymbol{\varepsilon}_{2: N}^{T}(t) P_{1} E^{2}\right) \boldsymbol{\varepsilon}_{2: N}(t+s) d s \\
\leq \tau(t) \boldsymbol{\varepsilon}_{2: N}^{T}(t) P_{1} E^{2} P_{1}^{-1}\left(P_{1} E^{2}\right)^{T} \boldsymbol{\varepsilon}_{2: N}(t) \\
\quad+\int_{-2 \tau(t)}^{-\tau(t)} \boldsymbol{\varepsilon}_{2: N}^{T}(t+s) P_{1} \boldsymbol{\varepsilon}_{2: N}(t+s) d s \\
\leq \tau \boldsymbol{\varepsilon}_{2: N}^{T}(t) P_{1} E^{2} P_{1}^{-1}\left(P_{1} E^{2}\right)^{T} \boldsymbol{\varepsilon}_{2: N}(t) \\
\quad+\int_{-2 \tau(t)}^{-\tau(t)} \boldsymbol{\varepsilon}_{2: N}^{T}(t+s) P_{1} \boldsymbol{\varepsilon}_{2: N}(t+s) d s,
\end{aligned}
$$

and let $a^{T}=-\boldsymbol{\varepsilon}_{2: N}^{T}(t) P_{1} E, b=F(t+s)$, and $\Phi=P_{1}^{-1}$; there is

$$
\begin{aligned}
-2 \boldsymbol{\varepsilon}_{2: N}^{T}(t) P_{1} E \int_{-\tau(t)}^{0} F(t+s) d s \\
\leq \tau(t) \boldsymbol{\varepsilon}_{2: N}^{T}(t) P_{1} E P_{1}^{-1}\left(P_{1} E\right)^{T} \varepsilon_{2: N}(t) \\
\quad+\int_{-\tau(t)}^{0} F^{T}(t+s) P_{1} F(t+s) d s \\
\leq \tau \boldsymbol{\varepsilon}_{2: N}^{T}(t) P_{1} E P_{1}^{-1}\left(P_{1} E\right)^{T} \varepsilon_{2: N}(t) \\
+\int_{-\tau(t)}^{0} F^{T}(t+s) P_{1} F(t+s) d s .
\end{aligned}
$$

Due to

$$
F(t)=\left(\begin{array}{c}
-\gamma\left(Q^{T} \otimes I_{m}\right) \Psi(\overline{\mathbf{x}}(t)) \\
0
\end{array}\right)
$$

then

$$
\begin{aligned}
\|F(t)\|^{2} & =\gamma^{2}\left\|\left(Q^{T} \otimes I_{m}\right) \Psi(\overline{\mathbf{x}}(t))\right\|^{2} \leq \gamma^{2} \bar{\rho}^{2}\|\overline{\mathbf{x}}(t)\|^{2} \\
& =\gamma^{2} \bar{\rho}^{2}\|e(t)\|^{2} \leq \gamma^{2} \bar{\rho}^{2}\|\boldsymbol{\varepsilon}(t)\|^{2},
\end{aligned}
$$

with the transformation $e(t)=\left(T^{T} \otimes I_{m}\right) \overline{\mathbf{x}}(t)$; we have

$$
\begin{aligned}
2 e_{1}^{T}(t) \dot{e}_{1}(t)= & -2 \gamma e_{1}^{T}(t)\left(\frac{\mathbf{1}_{N}^{T}}{\sqrt{N}} \otimes I_{m}\right) \Psi(\overline{\mathbf{x}}(t)) \\
= & -2 \gamma \overline{\mathbf{x}}^{T}(t) \Psi(\overline{\mathbf{x}}(t)) \\
& +2 \gamma e_{2}^{T}(t)\left(Q^{T} \otimes I_{m}\right) \Psi(\overline{\mathbf{x}}(t)) ;
\end{aligned}
$$

then, from Assumption 4, it follows that

$$
\begin{aligned}
2 \varepsilon_{2: N}^{T} & (t) P_{1} F+2 e_{1}^{T}(t) \dot{e}_{1}(t) \\
= & -2 \gamma\left(e_{2}^{T}(t)+\vartheta_{2}^{T}(t)\right)\left(Q^{T} \otimes I_{m}\right) \Psi(\overline{\mathbf{x}}(t)) \\
& -2 \gamma \overline{\mathbf{x}}^{T}(t) \Psi(\overline{\mathbf{x}}(t)) \\
& +2 \gamma e_{2}^{T}(t)\left(Q^{T} \otimes I_{m}\right) \Psi(\overline{\mathbf{x}}(t)) \\
= & -2 \gamma \vartheta_{2}^{T}(t)\left(Q^{T} \otimes I_{m}\right) \Psi(\overline{\mathbf{x}}(t)) \\
& -2 \gamma \overline{\mathbf{x}}^{T}(t) \Psi(\overline{\mathbf{x}}(t)) \\
\leq & \vartheta_{2}^{T}(t) \vartheta_{2}(t)+\gamma^{2}\left\|\left(Q^{T} \otimes I_{m}\right) \Psi(\overline{\mathbf{x}}(t))\right\|^{2} \\
& -2 \gamma \underline{m}\|\overline{\mathbf{x}}(t)\|^{2} \\
\leq & \vartheta_{2}^{T}(t) \vartheta_{2}(t)+\gamma^{2} \bar{\rho}^{2}\|\overline{\mathbf{x}}(t)\|^{2}-2 \gamma \underline{m}\|\overline{\mathbf{x}}(t)\|^{2} \\
\leq & \boldsymbol{\varepsilon}_{2: N}^{T}(t) \boldsymbol{\varepsilon}_{2: N}(t)-\left(2 \gamma \underline{m}-\gamma^{2} \bar{\rho}^{2}\right)\|\overline{\mathbf{x}}(t)\|^{2},
\end{aligned}
$$

where $\underline{m}=\min \left\{m_{1}, m_{2}, \ldots, m_{N}\right\}$ and $\bar{\rho}=\max \left\{\rho_{1}, \rho_{2}, \ldots\right.$, $\left.\rho_{N}\right\}$.

According to the Lyapunov-Razumikhin Theorem, take $\phi(s)=q s$ for some constant $q>1$. In case that

$$
V(\boldsymbol{\varepsilon}(t+\theta))<q V(\boldsymbol{\varepsilon}(t)), \quad \theta \in[-2 \tau, 0],
$$

then

$$
\begin{aligned}
& \boldsymbol{\varepsilon}_{2: N}^{T}(t+s) P_{1} \boldsymbol{\varepsilon}_{2: N}(t+s) \\
& \quad \leq e_{1}^{T}(t+s) e_{1}(t+s)+\boldsymbol{\varepsilon}_{2: N}^{T}(t+s) P_{1} \boldsymbol{\varepsilon}_{2: N}(t+s) \\
& \quad=\boldsymbol{\varepsilon}^{T}(t+s) P \boldsymbol{\varepsilon}(t+s)<q \boldsymbol{\varepsilon}^{T}(t) P \boldsymbol{\varepsilon}(t) .
\end{aligned}
$$

Next, considering the integral term in (38) and according to (40), we can obtain

$$
\int_{-\tau(t)}^{0} F^{T}(t+s) P_{1} F(t+s) d s
$$

$$
\begin{aligned}
& \leq \lambda_{\max }\left(P_{1}\right) \int_{-\tau(t)}^{0} F^{T}(t+s) F(t+s) d s \\
& \leq \lambda_{\max }\left(P_{1}\right) \gamma^{2} \bar{\rho}^{2} \int_{-\tau(t)}^{0} \boldsymbol{\varepsilon}^{T}(t+s) \boldsymbol{\varepsilon}(t+s) d s \\
& \leq \frac{\lambda_{\max }\left(P_{1}\right)}{\lambda_{\min }(P)} \gamma^{2} \bar{\rho}^{2} \int_{-\tau(t)}^{0} \boldsymbol{\varepsilon}^{T}(t+s) P \boldsymbol{\varepsilon}(t+s) d s \\
& \leq \delta \gamma^{2} \bar{\rho}^{2} q \int_{-\tau(t)}^{0} \boldsymbol{\varepsilon}^{T}(t) P \boldsymbol{\varepsilon}(t) d s \\
& \leq \delta \gamma^{2} \bar{\rho}^{2} q \tau\left[e_{1}^{T}(t) e_{1}(t)+\boldsymbol{\varepsilon}_{2: N}^{T}(t) P_{1} \boldsymbol{\varepsilon}_{2: N}(t)\right] \\
& \leq \delta \gamma^{2} \bar{\rho}^{2} q \tau \overline{\mathbf{x}}^{T}(t) \overline{\mathbf{x}}(t)+\delta \gamma^{2} \bar{\rho}^{2} q \tau \boldsymbol{\varepsilon}_{2: N}^{T}(t) P_{1} \boldsymbol{\varepsilon}_{2: N}(t) \\
& \leq \delta \gamma^{2} \bar{\rho}^{2} q \tau \boldsymbol{\varepsilon}^{T}(t) \boldsymbol{\varepsilon}(t)+\delta \gamma^{2} \bar{\rho}^{2} q \tau \boldsymbol{\varepsilon}_{2: N}^{T}(t) P_{1} \boldsymbol{\varepsilon}_{2: N}(t),
\end{aligned}
$$


and, substituting (44) into the integral term in (36), we can obtain

$$
\begin{aligned}
& \int_{-\tau(t)}^{0} \boldsymbol{\varepsilon}_{2: N}^{T}(t+s) P_{1} \boldsymbol{\varepsilon}_{2: N}(t+s) d s \\
& \quad< \int_{-\tau(t)}^{0} q \boldsymbol{\varepsilon}^{T}(t) P \boldsymbol{\varepsilon}(t) d s \\
& \quad< \int_{-\tau(t)}^{0}\left[q e_{1}^{T}(t) e_{1}(t)+q \boldsymbol{\varepsilon}_{2: N}^{T}(t) P_{1} \boldsymbol{\varepsilon}_{2: N}(t)\right] d s \\
& \quad<q \tau e_{1}^{T}(t) e_{1}(t)+q \tau \boldsymbol{\varepsilon}_{2: N}^{T}(t) P_{1} \boldsymbol{\varepsilon}_{2: N}(t) \\
& \quad<q \tau\|\overline{\mathbf{x}}(t)\|^{2}+q \tau \boldsymbol{\varepsilon}_{2: N}^{T}(t) P_{1} \boldsymbol{\varepsilon}_{2: N}(t) \\
& \quad \leq q \tau \boldsymbol{\varepsilon}^{T}(t) \boldsymbol{\varepsilon}(t)+q \tau \boldsymbol{\varepsilon}_{2: N}^{T}(t) P_{1} \boldsymbol{\varepsilon}_{2: N}(t) .
\end{aligned}
$$

Similarly,

$$
\begin{aligned}
& \int_{-2 \tau(t)}^{-\tau(t)} \boldsymbol{\varepsilon}_{2: N}^{T}(t+s) P_{1} \boldsymbol{\varepsilon}_{2: N}(t+s) d s \\
& \quad<q \tau \boldsymbol{\varepsilon}^{T}(t) \boldsymbol{\varepsilon}(t)+q \tau \boldsymbol{\varepsilon}_{2: N}^{T}(t) P_{1} \boldsymbol{\varepsilon}_{2: N}(t) .
\end{aligned}
$$

Then from (35) and above inequalities, we have

$$
\begin{aligned}
\dot{V}( & \boldsymbol{\varepsilon}(t)) \leq-\boldsymbol{\varepsilon}_{2: N}^{T}(t)\left(R-I_{2 N-2}\right) \boldsymbol{\varepsilon}_{2: N}(t)+\tau \boldsymbol{\varepsilon}_{2: N}^{T}(t) \\
& \cdot\left[P_{1} E C P_{1}^{-1}\left(P_{1} E C\right)^{T}+P_{1} E^{2} P_{1}^{-1}\left(P_{1} E^{2}\right)^{T}\right. \\
& \left.+P_{1} E P_{1}^{-1}\left(P_{1} E\right)^{T}+\left(2 q+\delta \gamma^{2} \bar{\rho}^{2} q\right) P_{1}\right] \boldsymbol{\varepsilon}_{2: N}(t) \\
& +\left(2 q \tau+\delta \gamma^{2} \bar{\rho}^{2} q \tau\right) \boldsymbol{\varepsilon}^{T}(t) \boldsymbol{\varepsilon}(t)-\left(2 \gamma \underline{m}-\gamma^{2} \bar{\rho}^{2}\right) \\
& \cdot\|\overline{\mathbf{x}}(t)\|^{2}
\end{aligned}
$$

where

$$
\begin{aligned}
R & =-\left(\bar{F}^{T} P_{1}+P_{1} \bar{F}\right)=\left(\begin{array}{cc}
(k-\alpha)\left(H^{T}+H\right) & I_{N-1} \\
I_{N-1} & 2 I_{N-1}
\end{array}\right) \\
& \otimes I_{m} \\
& P_{1} E C P_{1}^{-1}\left(P_{1} E C\right)^{T}+P_{1} E^{2} P_{1}^{-1}\left(P_{1} E^{2}\right)^{T} \\
& +P_{1} E P_{1}^{-1}\left(P_{1} E\right)^{T} \\
& =\left(\begin{array}{ll}
(k-\alpha)\left[(k+\alpha) H H^{T}+k^{3} H^{2}\left(H^{2}\right)^{T}\right] & 0 \\
0 & 0
\end{array}\right) \\
& \otimes I_{m} .
\end{aligned}
$$

According to Lemma 3, if $k$ satisfies condition (21), then $R-$ $I_{2 N-2}$ is positive definite; we have

$$
\begin{aligned}
\boldsymbol{\varepsilon}_{2: N}^{T}(t) & \left(R-I_{2 N-2}\right) \boldsymbol{\varepsilon}_{2: N}(t) \geq \underline{\lambda}_{2} \boldsymbol{\varepsilon}_{2: N}^{T}(t) \boldsymbol{\varepsilon}_{2: N}(t) \\
= & \underline{\lambda}_{2} \boldsymbol{\varepsilon}_{2: N}^{T}(t) \boldsymbol{\varepsilon}_{2: N}(t)+\underline{\lambda}_{2} e_{1}^{T}(t) e_{1}(t) \\
& \quad-\underline{\lambda}_{2} e_{1}^{T}(t) e_{1}(t)=\underline{\lambda}_{2} \boldsymbol{\varepsilon}^{T}(t) \boldsymbol{\varepsilon}(t)-\underline{\lambda}_{2} e_{1}^{T}(t) e_{1}(t) \\
\geq & \underline{\lambda}_{2} \boldsymbol{\varepsilon}^{T}(t) \boldsymbol{\varepsilon}(t)-\underline{\lambda}_{2}\|\overline{\mathbf{x}}(t)\|^{2},
\end{aligned}
$$

if condition (20) is satisfied and due to the fact that $\boldsymbol{\varepsilon}_{2: N}^{T}(t) \boldsymbol{\varepsilon}_{2: N}(t) \leq \boldsymbol{\varepsilon}^{T}(t) \boldsymbol{\varepsilon}(t)$; then

$$
\begin{aligned}
\dot{V} & (\boldsymbol{\varepsilon}(t)) \leq-\underline{\lambda}_{2} \boldsymbol{\varepsilon}^{T}(t) \boldsymbol{\varepsilon}(t)+\underline{\lambda}_{2}\|\overline{\mathbf{x}}(t)\|^{2}+\tau \boldsymbol{\varepsilon}_{2: N}^{T}(t) \\
\cdot & \left\{(k-\alpha)\left[(k+\alpha) \bar{\lambda}_{1}+k^{3} \bar{\lambda}_{2}\right]+\left(2 q+\delta \gamma^{2} \bar{\rho}^{2} q\right) \bar{\mu}\right\} \\
& \cdot \boldsymbol{\varepsilon}_{2: N}(t)+2\left(\delta \gamma^{2} \bar{\rho}^{2} q \tau+q \tau\right) \boldsymbol{\varepsilon}^{T}(t) \boldsymbol{\varepsilon}(t)-(2 \gamma \underline{m} \\
& \left.-\gamma^{2} \bar{\rho}^{2}\right)\|\overline{\mathbf{x}}(t)\|^{2} \leq-\underline{\lambda}_{2} \boldsymbol{\varepsilon}^{T}(t) \boldsymbol{\varepsilon}(t)+\tau \boldsymbol{\varepsilon}^{T}(t) \\
& \cdot\left\{(k-\alpha)\left[(k+\alpha) \bar{\lambda}_{1}+k^{3} \bar{\lambda}_{2}\right]+\left(2 q+\delta \gamma^{2} \bar{\rho}^{2} q\right) \bar{\mu}\right. \\
& \left.+\left(2 q+\delta \gamma^{2} \bar{\rho}^{2} q\right)\right\} \boldsymbol{\varepsilon}(t)-\left(2 \gamma \underline{m}^{2}-\gamma^{2} \bar{\rho}^{2}-\underline{\lambda}_{2}\right) \\
& \cdot\|\overline{\mathbf{x}}(t)\|^{2} \leq-\underline{\lambda}_{2} \boldsymbol{\varepsilon}^{T}(t) \boldsymbol{\varepsilon}(t)+\tau \boldsymbol{\varepsilon}^{T}(t) \\
& \cdot\left\{(k-\alpha)\left[(k+\alpha) \bar{\lambda}_{1}+k^{3} \bar{\lambda}_{2}\right]\right. \\
& \left.+\left(2 q+\delta \gamma^{2} \bar{\rho}^{2} q\right)(\bar{\mu}+1)\right\} \boldsymbol{\varepsilon}(t)
\end{aligned}
$$

and we take $\tau$ has the upper bound in (22); then $\dot{V}(\boldsymbol{\varepsilon}(t))$ is negative definite. Thus by the Lyapunov-Razumikhin theorem, we can conclude that $\varepsilon(t) \rightarrow 0$; that is, $e(t) \rightarrow 0_{m N}, \vartheta(t) \rightarrow$ $0_{m N}$ as $t \rightarrow \infty$.

With the transformation $\overline{\mathbf{x}}(t)=\left(T \otimes I_{m}\right) e(t)$ and $\overline{\mathbf{w}}(t)=$ $\left(T \otimes I_{m}\right) \vartheta(t)$ and $T$ is a orthogonal matrix, we can obtain $\overline{\mathbf{x}}(t) \rightarrow 0_{m \mathrm{~N}}, \overline{\mathbf{w}}(t) \rightarrow 0_{m N}$, which means $\mathbf{x}(t) \rightarrow \mathbf{x}^{*}, \mathbf{w}(t) \rightarrow$ $\mathbf{w}^{*}$ as $t \rightarrow \infty$. As a result, this proof is completed.

Remark 9. The continuous-time protocol considered in this paper is based on the algorithm proposed in [15], and under the same communication topology, but the conditions of convergence analysis needed by this paper are more relaxed. From (20) and (21), it is clearly shown that $k^{*}$ is independent of parameters $\beta$ and $\gamma$ but dependent on $\alpha$ and communication topology, while $\tau^{*}$ is independent of constant $m_{i}$ in this paper compared to [15]. We can know when the number of the agents is large, it would be difficult to verify the LMI condition, but, in this paper, it only needs the model parameters to meet some boundary conditions, and when considering the dynamic system with time-varying delay, the Lyapunov function with Razumikhin technique is also an effective method compared to Lyapunov-Krasovskii method.

\section{Simulations}

In this section, we give an example to validate our theoretical results. In the example, we consider a multiagent system 


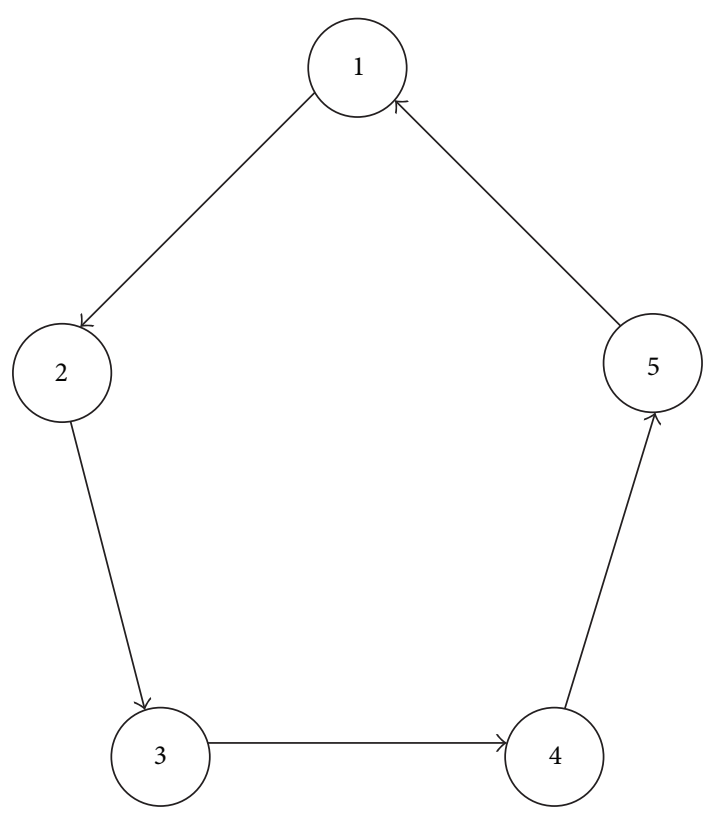

Figure 1: Connected graph.

consisting of five agents. Suppose that the interconnected topology is described as in Figure 1.

Consider the following optimization problem:

$$
\text { minimize } f(x)=\sum_{i=1}^{N} f_{i}(x), \quad x \in \mathscr{R},
$$

where the local objective function is given as follows:

$$
\begin{aligned}
& f_{1}(x)=0.7(x-8)^{2}, \\
& f_{2}(x)=0.6 x^{2}-2, \\
& f_{3}(x)=(x+2)^{2}, \\
& f_{4}(x)=0.8 x^{2}+2 x \\
& f_{5}(x)=\sin \frac{x}{2}+\frac{x^{2}}{2} .
\end{aligned}
$$

Obviously, for $i=1,2, \ldots, 5, f_{i}$ is differentiable and satisfies Assumption 4. Choosing $\alpha=0.6, k=2.2, \gamma=0.4, q=1.1$ and time-varying delay $\tau(t)=0.01|\cos (t)|$, we can obtain $\bar{\rho}=$ $2, m=1, k^{*}=2.0472$ and $\tau^{*}=0.0004$.

Let the initial values $x(0)=\left[x_{1}(0), x_{2}(0), x_{3}(0), x_{4}(0)\right.$, $\left.x_{5}(0)\right]^{T}=[-8,-2,2,3,10]^{T}, w(0)=\left[w_{1}(0), w_{2}(0), w_{3}(0)\right.$, $\left.w_{4}(0), w_{5}(0)\right]^{T}=[0,0,0,0,0]^{T}$. The simulation results are shown in Figures 2 and 3.

We can see that the trajectories $x_{i}$ of each agent $i$ converge to the global optimal solution $x^{*}=0.6565$ of the objective function $f(x)=\sum_{i=1}^{N} f_{i}(x)$ and all the trajectories $w_{i}$ converge to a constant, respectively, for $i=1,2, \ldots, 5$. The optimal value of $f(x)$ is 45.2602 .

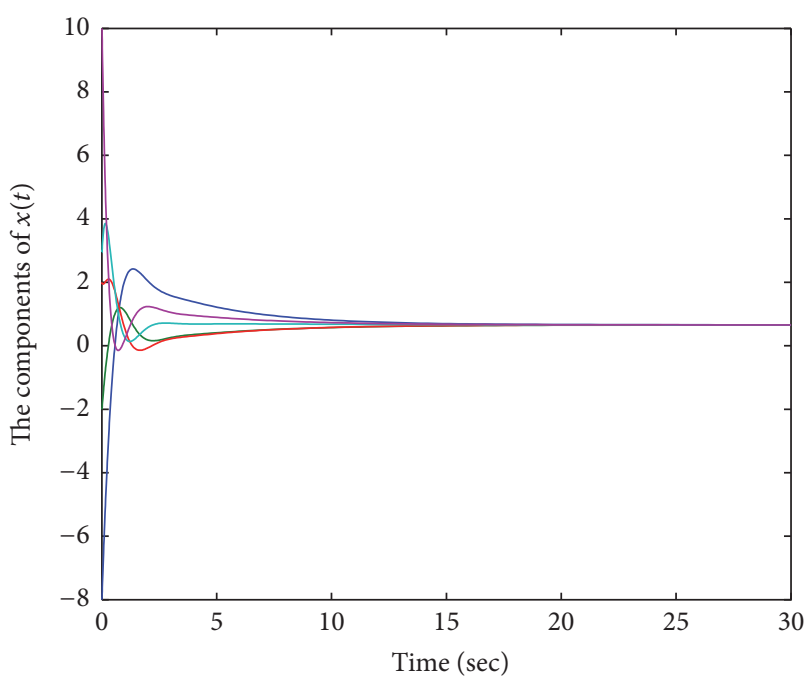

FIgURE 2: The trajectories of $x_{i}$.

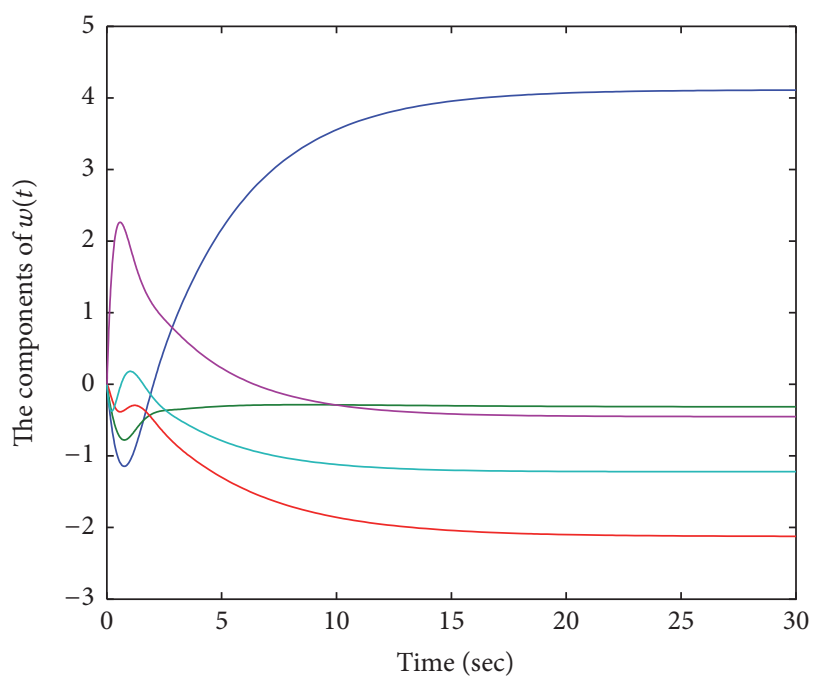

FIgURE 3: The trajectories of $w_{i}$.

\section{Conclusion}

In this paper, the consensus optimization problem of multiagents with communication delays was considered. By a continuous-time algorithm, consensus and optimization under some parameter bound conditions are ensured. Graph theory is used to describe the interconnection topologies. Lyapunov-Razumikhin theory were employed for stability analysis. The connectivity assumption of directed graph plays a key role in the analysis of algorithm convergence. Numerical examples were given to illustrate the theoretical results.

\section{Conflicts of Interest}

The authors declare that they have no conflicts of interest. 


\section{Acknowledgments}

This work is supported in part by Natural Science Foundation of China (61273183 and 61374028).

\section{References}

[1] P. Yi, Y. Hong, and F. Liu, "Distributed gradient algorithm for constrained optimization with application to load sharing in power systems," Systems \& Control Letters, vol. 83, pp. 45-52, 2015.

[2] T.-H. Chang, A. Nedić, and A. Scaglione, "Distributed constrained optimization by consensus-based primal-dual perturbation method," IEEE Transactions on Automatic Control, vol. 59, no. 6, pp. 1524-1538, 2014.

[3] M. Rabbat and R. Nowak, "Distributed optimization in sensor networks," in Proceedings of the 3rd International Symposium on Information Processing in Sensor Networks (IPSN '04), pp. 20-27, IEEE, April 2004.

[4] S. S. Ram, V. V. Veeravalli, and A. Nedić, "Distributed nonautonomous power control through distributed convex optimization," in Proceedings of the 28th Conference on Computer Communications (IEEE INFOCOM '09), pp. 3001-3005, IEEE, Rio de Janeiro, Brazil, April 2009.

[5] R. Olfati-Saber and R. M. Murray, "Consensus problems in networks of agents with switching topology and time-delays," IEEE Transactions on Automatic Control, vol. 49, no. 9, pp. 15201533, 2004.

[6] R. Olfati-Saber, J. A. Fax, and R. M. Murray, "Consensus and cooperation in networked multi-agent systems," Proceedings of the IEEE, vol. 95, no. 1, pp. 215-233, 2007.

[7] J. Hu and Y. S. Lin, "Consensus control for multi-agent systems with double-integrator dynamics and time delays," IET Control Theory \& Applications, vol. 4, no. 1, pp. 109-118, 2010.

[8] W. X. Li and Z. W. Chen, "Leader-following consensus of second-order multi-agent systems with time-delay and nonlinear dynamics," in Proceedings of the 31th Chinese Control Conference, pp. 7251-7256, 2015.

[9] A. Nedić and A. Ozdaglar, "Distributed subgradient methods for multi-agent optimization," IEEE Transactions on Automatic Control, vol. 54, no. 1, pp. 48-61, 2009.

[10] A. Nedic, A. Ozdaglar, and P. A. Parrilo, "Constrained consensus and optimization in multi-agent networks," IEEE Transactions on Automatic Control, vol. 55, no. 4, pp. 922-938, 2010.

[11] I. Lobel and A. Ozdaglar, "Distributed subgradient methods for convex optimization over random networks," IEEE Transactions on Automatic Control, vol. 56, no. 6, pp. 1291-1306, 2011.

[12] M. Zhu and S. Martinez, "On distributed convex optimization under inequality and equality constraints," IEEE Transactions on Automatic Control, vol. 57, no. 1, pp. 151-164, 2012.

[13] J. Wang and N. Elia, "A control perspective for centralized and distributed convex optimization," in Proceedings of the 50th IEEE Conference on Decision and Control and European Control Conference (CDC-ECC '11), pp. 3800-3805, IEEE, Orlando, Fla, USA, December 2011.

[14] S. S. Kia, J. Cortés, and S. Martínez, "Distributed convex optimization via continuous-time coordination algorithms with discrete-time communication," Automatica, vol. 55, pp. 254264, 2015.

[15] S. F. Yang, Q. S. Liu, and J. Wang, "Distributed optimization based on a multiagent system in the presence of communication delays," IEEE Transactions on Systems, Man, and Cybernetics: Systems, 2016.

[16] B. Gharesifard and J. Cortes, "Distributed continuous-time convex optimization on weight-balanced digraphs," IEEE Transactions on Automatic Control, vol. 59, no. 3, pp. 781-786, 2014.

[17] Q. Liu and J. Wang, "A second-order multi-agent network for bound-constrained distributed optimization," IEEE Transactions on Automatic Control, vol. 60, no. 12, pp. 3310-3315, 2015.

[18] Y. Zhang and Y. Hong, "Distributed optimization design for high-order multi-agent systems," in Proceedings of the 34th Chinese Control Conference (CCC '15), pp. 7251-7256, IEEE, July 2015.

[19] J. Lu and C. Y. Tang, "Zero-gradient-sum algorithms for distributed convex optimization: the continuous-time case," IEEE Transactions on Automatic Control, vol. 57, no. 9, pp. 23482354, 2012.

[20] J. W. Wang and J. X. Li, "Exponential consensus of second-order multi-agent systems with time-varying delay under switching network," in Proceedings of the IEEE International Conference on Information and Automation, pp. 1058-1063, IEEE, August 2015.

[21] Y. G. Sun, L. Wang, and G. M. Xie, "Average consensus in networks of dynamic agents with switching topologies and multiple time-varying delays," Systems \& Control Letters, vol. 57, no. 2, pp. 175-183, 2008.

[22] C. Godsil and G. Royle, Algebraic Graph Theory, Springer, New York, NY, USA, 2001.

[23] S. Boyd, L. E. Ghaoui, E. Feron, and V. Balakrishnan, Linear Matrix Inequalities in System and Control Theory, SIAM, Philadelphia, Pa, USA, 1994.

[24] R. T. Rockafellar, Convex Analysis, Princeton University Press, Princeton, NJ, USA, 1972.

[25] J. K. Hale and S. M. V. Lunel, Introduction to the Theory of Functional Differential Equations, Springer, New York, NY, USA, 1991. 


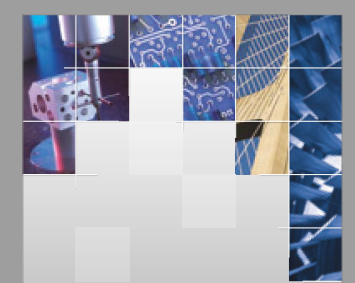

\section{Enfincering}
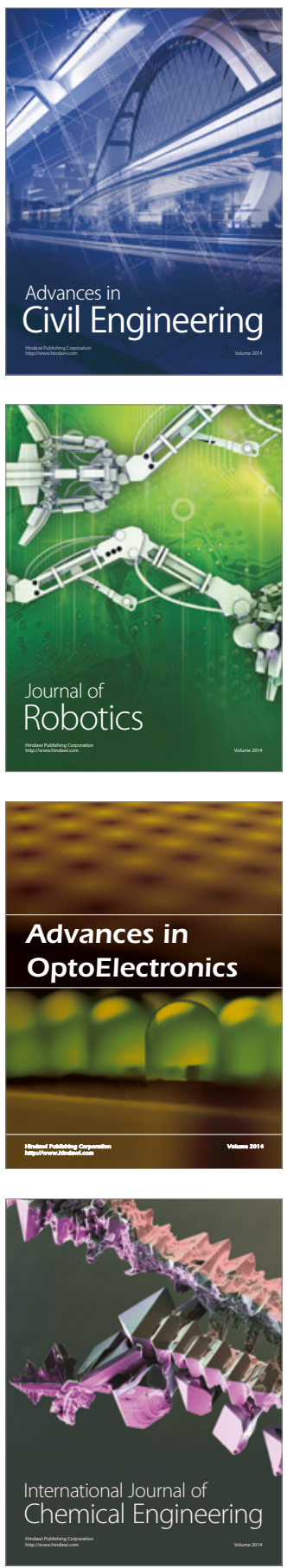

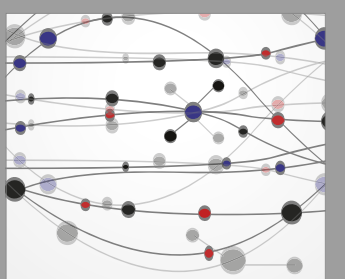

The Scientific World Journal

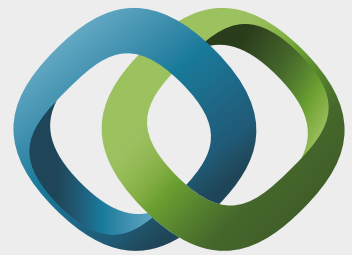

\section{Hindawi}

Submit your manuscripts at

https://www.hindawi.com
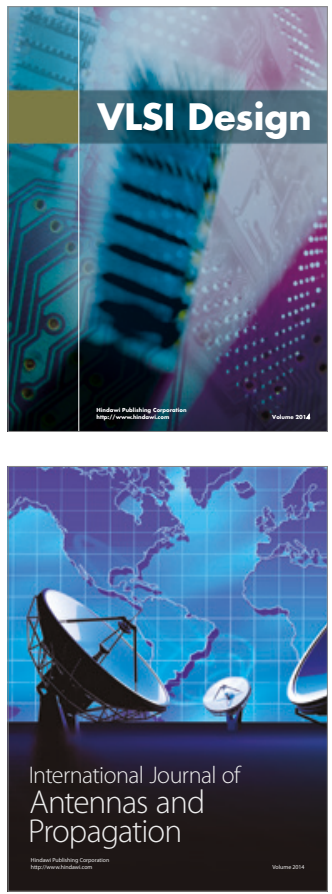

\section{Rotating}

Machinery
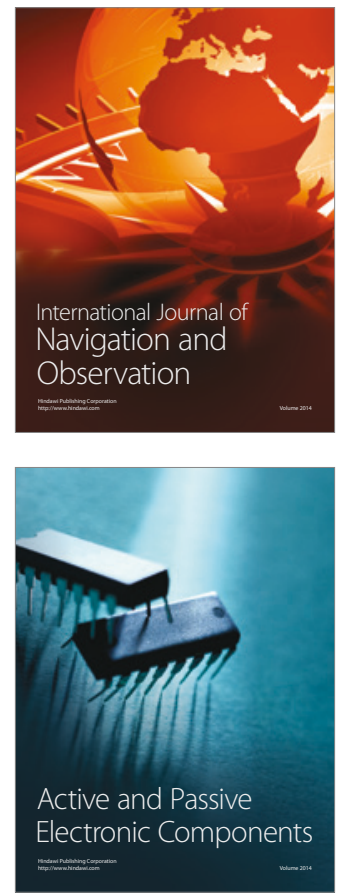
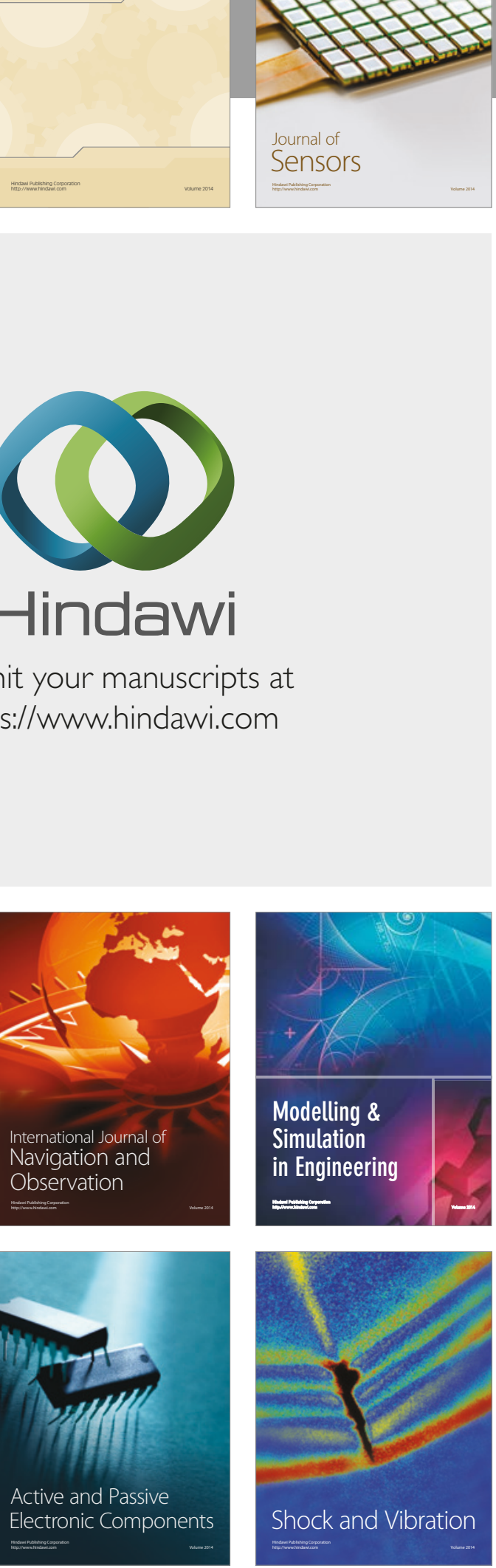
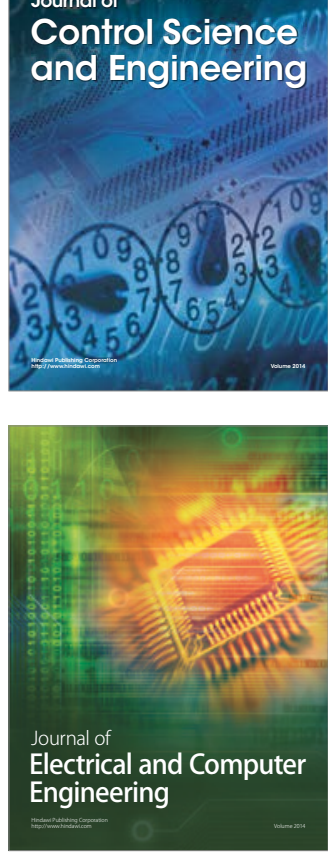

Distributed

Journal of

Control Science

and Engineering
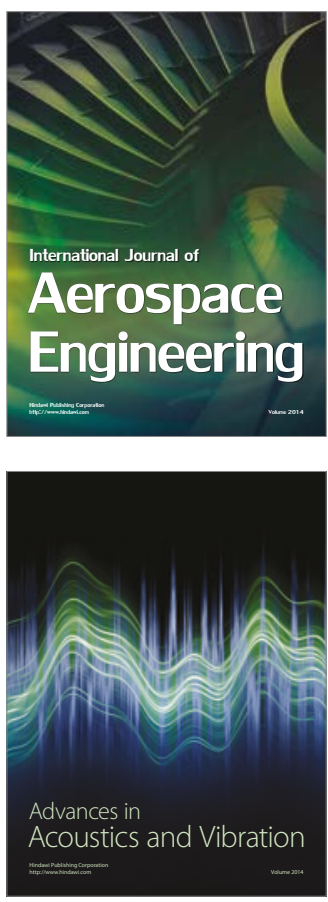

Sensor Networks 